\title{
The study of the feasibility of segmental bone defect repair with tissue- engineered bone membrane: a qualitative observation
}

\author{
Lin Zhao · Jun-Li Zhao $\cdot$ Lin Wan • \\ Shuan-Ke Wang
}

Received: 20 July 2007 / Accepted: 19 February 2008/Published online: 12 April 2008

(C) Springer-Verlag 2008

\begin{abstract}
The objective of the study was to investigate the feasibility of intramembranous osteogenesis from tissue-engineered bone membrane in vivo. Bone marrow mesenchymal stem cells (MSCs) of rabbits were harvested, expanded and some of them were induced into osteoblasts. Porcine small intestinal submucosa (SIS) was converted by a series of physical and chemical procedures into a scaffold. MSCs and induced osteoblasts were seeded separately onto the scaffold, thus fabricating two kinds of tissueengineered bone membrane. A total of 12 New Zealand rabbits were subjected to a surgical operation; a $15 \mathrm{~mm}$ bone segment, including the periosteum, was resected from the radius on both sides of each rabbit to create critical bone defects. The two kinds of tissue-engineered bone membrane and SIS (as control) were implanted randomly into the site of bone defect. The animals had radiographs and were killed after 4 weeks. The specimens were harvested and histological examination performed for evidence of osteogenesis. Bone tissue had formed in defects treated by the two kinds of tissue-engineered bone membrane at 4 weeks. This was supported by the X-ray and histological examination, which confirmed the segmental gap bridged by bone. There was no attempt to bridge in the bone defect treated by SIS. Tissue-engineered bone membrane, constructed by seeding allogeneic cells on
\end{abstract}

L. Zhao $(\bowtie) \cdot$ L. Wan · S.-K. Wang

Orthopaedic Institute of the 2nd Hospital of Lanzhou University, 80 CuiYingMen, ChengGuan District, 730030 Lanzhou City, People's Republic of China

e-mail: bonezl@sina.com.cn

J.-L. Zhao

Department of Nephrology, The 2nd Hospital of Lanzhou

University, 80 CuiYingMen, ChengGuan District,

730030 Lanzhou City, People's Republic of China an xenogeneic and bio-derived scaffold, can repair critical bone defects successfully.

Keywords Osteogenesis - Tissue engineering · Mesenchymal stem cells · Stem cell transplantation . Osteogenic membrane

\section{Introduction}

Although bone possesses the capacity for regenerative growth and remodelling, both processes become impaired in clinical situations in which excessive bone loss is caused by disease, trauma or through tumour resection [1]. In order to address the need of an increasing number of patients who require bone for skeletal reconstruction, surgeons can overcome the disadvantages linked to auto- or allografts by choosing the tissue engineering approach $[2,3]$. Tissue engineering for bone faces the challenge of exporting successful laboratory protocols; the transfer of such technology for widespread clinical use is still impractical.

Traditional research in bone tissue engineering describes the use of autogenous cells as the seeding cells because of concerns over immunogenicity $[4,5]$. This is impractical for extensive clinical application as it confines the tissue engineering process to an individual therapy and thereby restricts the possible future industrialization and standardization of tissue-engineered products. In addition, an individualized procedure starting with the proliferation and construction of autogenous cells in vitro would be difficult to satisfy the needs of tissue in emergency clinical situations.

Bio-derived materials and synthetic polymers made into 3-D porous structures possess some rigidity and have 
served as scaffolds in most traditional bone tissue engineering experiments [6-10]. However, these synthetic derivatives do not mimic a structure similar to bone tissue in vivo. These 3-D porous scaffolds are difficult to seed cells beyond the outer surface, especially when present in large volume composites. Furthermore, the superficially seeded cells survive only through nutritional diffusion and in so doing there is a limit to the engineered bone tissue arising from these scaffolds of about less than $0.5 \mathrm{~mm}$ thick [11], which is not of much value for clinical practice [12]. Therefore, these large 3-D scaffolds become a spatial hindrance for bone regeneration.

Natural bone tissue is highly vascularized and relies on blood vessels to deliver nutrients and oxygen to cells deep in the mineralized bone matrix. One of the greatest challenges faced in bone tissue engineering is how to enhance the supporting scaffold and newly formed bone tissue into establishing a natural capillary network [13]. The success of regenerating large volumes of bone, which is valuable for clinical use, depends on vascularization of these grafts. This still remains the greatest challenge for tissue engineering of bone, the ability to revascularize the graft.

The bone develops through two methods of osteogenesis: endochondral and intramembranous. Intramembranous osteogenesis has been shown to be important in bone fracture healing and bone defect repairing. Although there are many successful studies of bone defect repair through autogeneic periosteum transplantation [14-16], there are only a few reports on the bridging of bone defects by tissue-engineered bone membrane [17]. A tissue-engineered bone membrane may potentially regenerate a large segment bone through the intramembranous route and this would circumvent the problem of inducing a new vascular system, as this form of osteogenesis induces simultaneous new bone formation and angiogenesis.

Of the several kinds of osteogenic cells, bone marrow mesenchymal stem cells (MSCs) seem to be the optimal seeding cells for bone tissue engineering owing to their ease of isolation, capacity of high replication and osteoblast differentiation [18-20]. Recent studies have reported that MSCs have the potential of allogeneic implantation as these cells do not express the molecules of MHC (major histocompatibility complex) and can adjust to the immune system of the recipient [18, 21-24]. Therefore, selection of MSCs as seeding cells in the construction of tissue-engineered bone membrane would appear a suitable choice, especially if the graft was used in an allogeneic recipient.

Porcine small intestinal submucosa (SIS) is a bioderived material, which has been shown to have low immunogenicity in more than 1,000 inter-species implantations [25]. It is also reported that SIS can enhance angiogenesis through releasing vascular endothelial growth factor (VEGF) during biodegradation in vivo [26]. There are several structural characteristics in SIS that are similar to periosteum, which make it optimal for use as a scaffold in tissue-engineered bone membrane viz., its elastic texture, biodegradability and thinness $[26,27]$. The membrane structure provides a large area for cells to attach and could permit cell survival through nutritional diffusion in the early stages before intrinsic angiogenesis. In addition, the membrane can guide the process of bone regeneration $[28,29]$.

\section{Materials and methods}

Porcine small intestinal submucosa (SIS) was harvested from the small intestine of healthy pigs within $4 \mathrm{~h}$ after killing. The procedure of processing SIS was in accordance with published protocols [26-28]. The submucosa layer was detached from the serous coat and muscular layers by blunt dissection and then cleaned by washing continuously with $40^{\circ} \mathrm{C}$ water. This submucosa layer was then treated with a series of chemical procedures at room temperature with the volume ratio between the submucosa matrix and liquid used below being 1:100 $(v / v t)$. The submucous membrane was submerged in $100 \mathrm{mmol} / \mathrm{L}$ EDTA and $10 \mathrm{mmol} / \mathrm{L} \mathrm{NaOH}(\mathrm{pH} 11-12)$ for $16 \mathrm{~h}$; deionized water was used to rinse until clear. The membrane was then soaked in a mixture of $1 \mathrm{mmol} / \mathrm{L} \mathrm{HCl}$ and $1 \mathrm{mmol} / \mathrm{L} \mathrm{NaCl}$ (pH $0-1)$ for $6 \sim 8 \mathrm{~h}$. Rinsing with deionized water was repeated and subsequent immersion was in $1 \mathrm{mmol} / \mathrm{L} \mathrm{NaCl}$ dissolved with PBS for $16 \mathrm{~h}$, followed by rinsing with deionized water. It was then dipped into PBS ( $\mathrm{pH} 7-7.4$ ) for $2 \mathrm{~h}$ and further rinsed with deionized water for $2 \mathrm{~h}(\mathrm{pH}$ 5.8-7.0). The submucous membrane was then soaked into $0.1 \%$ peroxyacetic acid and $20 \%$ ethanol for $8 \mathrm{~h}$, rinsed with $0.05 \%$ sodium azide (Sigma) dissolved with PBS for $2 \mathrm{~h}$ and freeze dried under $-70^{\circ} \mathrm{C}$ with lyophilize r(HETOHOL Power Dry LL3000) for more than $8 \mathrm{~h}$. It was then packaged in a plastic cover and irradiated (25-35 kGy) under $\mathrm{Co}_{60} \gamma$-ray for half an hour. The SIS products were stored in a $-80^{\circ} \mathrm{C}$ refrigerator for later use.

Isolation, cultivation and osteogenic differentiation and identification of rabbit-derived MSCs

Bone marrow aspirates were harvested in heparin $(200 \mathrm{U} / \mathrm{mL}$ total) from the femoral medullary canal of healthy neonatal (less than 10 days old) New Zealand rabbits. The cell culture was processed according to a modified protocol $[5,6]$. The aspirates were mixed with equal volumes of Dulbecco's Modified Eagles Medium (DMEM, Gibco) supplemented with $10 \%$ fetal bovine serum (FBS, Gibco) and centrifuged at 1,000 rpm for $10 \mathrm{~min}$. The fat layer and supernatant were removed. The recovered cells were 
re-suspended in $5 \mathrm{~mL}$ DMEM containing $10 \%$ FBS and layered over with Percoll (Pharmacia) at a density of $1.073 \mathrm{~g} / \mathrm{L}$ and then centrifuged at 3,000 rpm for $30 \mathrm{~min}$. The middle layer of granules were removed carefully and washed with PBS at 1,000 rpm centrifugation twice for 5 min and finally re-suspended in DMEM supplemented with $10 \%$ FBS, penicillin $\mathrm{G}(100 \mathrm{U} / \mathrm{mL})$ and streptomycin sulphate $(100 \mathrm{mcg} / \mathrm{mL})$. Cellular cultures were started at a cellular concentration of $2 \times 10^{5} / \mathrm{mL}$ in $25 \mathrm{~cm}^{2}$ flasks and maintained at $37^{\circ} \mathrm{C}$ in a humidified $5 \%$ carbon dioxide environment. When the cultures reached $90 \%$ of confluence, the cells were passaged. On passaging, the cells were detached from the flask by incubation with a mixture of an enzyme solution of $0.125 \%$ trypsin(Gibco) and $0.02 \%$ ethylenediaminetetraacetic acid (EDTA, Gibco) for 2-5 min at room temperature.

Part of the MSCs from passage 3 were stimulated for 1 week in a standard medium supplemented with $50 \mathrm{mg} / \mathrm{L}$ ascorbic acid (Sigma), $10 \mathrm{mmol} / \mathrm{L} \quad \beta$-glycerophosphate (Sigma) and $10^{-8} \mathrm{~mol} / \mathrm{L}$ dexamethasone (Sigma).

Induced MSCs were harvested with the mixture of enzymes mentioned above and transferred to sterile slides for cellular adherence. The cells on the slides were fixed for more than $72 \mathrm{~h}$ with $4 \%$ paraformaldehyde or cool acetone at $4^{\circ} \mathrm{C}$, respectively. The former was used as a specimen for immunocytochemistry and the latter for alkaline phosphatise (ALP) detection. The specimens for immunocytochemistry were stained using osteocalcin and a collagen type I immunohistochemistry detection kit (Boster, Wuhan, China) according to routine procedures; ALP detection was carried out using ALP's Gomori staining.

\section{Preparation of implants}

To construct bone membrane implants, we choose MSCs and osteoblasts induced from the MSCs described above as the osteogenetic seeding cells. These two types of seeding cells were combined with SIS, which ubiquitously served as the prefabricated scaffold, thereby producing two kinds of tissue-engineered bone membrane: namely, tissue-engineered bone membrane (M1) constructed by MSCs and SIS, and tissue-engineered bone membrane (M2 ) constructed by osteoblasts (induced from MSCs) and SIS. The procedure of combining seeding cells and the scaffold will be described briefly. First, the SIS was clipped into a size of $2 \mathrm{~cm} \times 2 \mathrm{~cm}$ and spread on the bottom of wells of a 6-well plaque and sterilized again under ultraviolet for more than an hour before being immersed overnight into DMEM containing 20\% FBS. The seeding cells were detached from the culture flasks with co-enzymes and the cellular concentration adjusted to $2 \times 10^{6} / \mathrm{mL}$ with DMEM containing $10 \%$ FBS. A
$200 \mu \mathrm{L}$ cellular suspension was applied onto the surface of the scaffold in each well. The culture of cells and scaffold were gently agitated in the incubator and left for $6 \mathrm{~h}$ until more culture medium was added. The incubation period lasted for 7 days before in vivo implantation was performed.

\section{Scanning electron microscopy (SEM)}

Some of the tissue-engineered bone membrane implants, which were cultured for 5 days were removed from the 6-well plaque and washed twice with PBS before being fixed with $2.5 \%$ neutral glutaraldehyde for scanning electron microscopy (SEM JSW-680LA, Japan) inspection. The surface of these implants was checked by SEM to ensure that the seeding cells adhered onto the scaffold abundantly.

Animals and operative procedures

Twelve 2-month-old New Zealand rabbits, $2.0-3.0 \mathrm{~kg}$ in weight, and purchased from the Animal Experiment Centre of the Lanzhou Institute of Biological Products (Lanzhou city, Gansu province, China) and approved by the Associate of Experiment Animal of Gansu Province were used in the study. All experiments were conducted with strict observation of institutional guidelines for the care and use of laboratory animals.

The rabbits were anaesthetized with an intraperitoneal injection of $3 \%$ pentobarbital solution $(40 \mathrm{mg} / \mathrm{kg}$ body weight) before surgery. Under sterile conditions, both sides of the mid-shaft of the radius was exposed through a lateral longitudinal skin incision and the forearm muscles divided. A $1.5 \mathrm{~cm}$ long section of the diaphysis was removed together with the periosteum using a cutting saw, thereby creating a segmental bone defect. The animals were then divided randomly into three groups:

1. group A - defect covered with M1;

2. group B - defect covered with $\mathrm{M} 2$ and

3. group C - defect covered with SIS.

All the prepared implants were wrapped snug around the ends of the bone defect and the edges of the bone membrane sutured with a 7-0 atraumatic suture. This converted the flat implant into a tubular membrane, which bridged across the ends of the bone defect. The incision was closed with 1-0 nylon suture. The forearms of the rabbits were immobilized with external fixators; antibiotics were injected routinely for the first 5 days post-surgery. The animals were reared separately for 4 weeks. All animals were killed by aeroembolism through intravenous air injection at 4 weeks and the specimens in the middle area of the defect were excised for histological examination. 
Experimental detection

X-ray radiography

All animals were radiographed (DR3000 Dryview8900, Koda, Japan) at 4 weeks after the external fixators were removed. The anteroposterior views of the radius and ulna from both sides of each animal were obtained by X-ray and the bone formation evaluated.

\section{Histological examination}

For histological examination, samples were fixed with $4 \%$ paraformaldehyde in a $0.1 \mathrm{M}$ phosphate buffer $(\mathrm{pH} 7.4)$ for 3 days. Following fixation, the samples were decalcified with $10 \%$ EDTA-2Na solution for 4 weeks at $4^{\circ} \mathrm{C}$. After demineralisation, the specimens were dehydrated through an ascending ethanol series and mounted in paraffin. Sections $5 \mu \mathrm{m}$ thick were obtained and stained with haematoxylin-eosin and Masson to observe new bone formation under microscopy.

\section{Results}

Cell and scaffold and implant

The MSCs we cultured showed a uniform fibroblast-like appearance as well as high proliferating potential (Fig. 1). The cellular components resembled the stem cells described in the literature. Although 1 week of osteogenic induction cannot change the MSCs into a typical osteoblast appearance, the induced MSCs did show some osteogenic differentiation phenotype with a high expression of ALP,

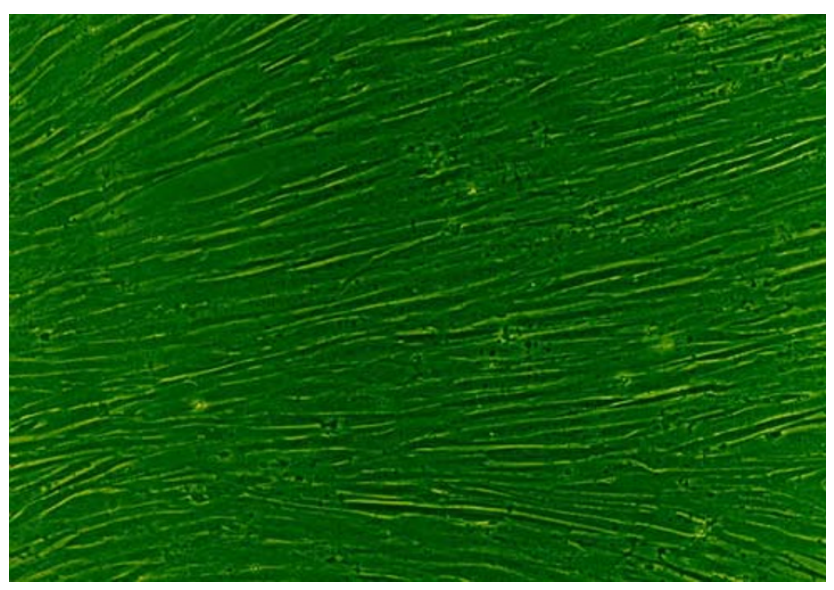

Fig. 1 Cultured rabbit MSCs showed a classical, long fusiform shape and a high proliferating potential that caused them to easily undergo confluence collagen type I and osteocalcin content intracellularly (Fig. 2). These findings suggested that the MSCs had already differentiated into osteoblasts functionally.

The SIS scaffold we manufactured was a white coloured membrane-like structure with a $100 \pm 20 \mu \mathrm{m}$ thickness and a flexible texture similar to natural bone membrane (Fig. 3). SEM observation revealed that the SIS had a velvet-like surface and irregular porous membrane structure constituted by mesh-like collagen fibre bundles (Fig. 4). The characteristics (thickness and porosity) of SIS were such that it was feasible to fabricate a tissue-engineered bone membrane implant. This scaffold not only provided a large cellular adherence area but also had the potential to allow the seeded cells to survive through diffusion of the nutrients from outside the scaffold owing to a thickness of less than $0.5 \mathrm{~mm}$ [11]. SEM observation of the surface of the tissue-engineered bone membrane showed that the seeded cells adhered to the SIS extensively with almost all of the scaffold surface occupied (Fig. 5).

X-ray evaluation

All defects were clearly discernible with no bone formation observed after 4 weeks at sites treated only with SIS (Fig. 6). This contrasts with the radio-opaque tissue observed at the same time at all sites treated with the M1 or M2 samples. The observed new bone density was lower than that at the edges of the defect and of cortical bone but higher than that of the olecranon process and medullary cavity. The defect treated with M1 or M2 were almost completely bridged and occupied by a newly formed, uniform density callous. Neither hypertrophy nor atrophy was observed on both ends of the bone defect.

\section{Histological observation}

Histological sections showed fibrous connective tissue and SIS degraded remnants filling in the region of defect repaired with SIS alone with no discernable osseous tissue. In comparison, newly formed osseous tissue was detected in the defect region treated with M1 or M2. The new bone tissue grew around irregular spherical porosities, which were occupied partially by medullary cavities or vessels in addition to degraded remnants of SIS (Fig. 7). No inflammatory cells were identified in all sections from all groups.

\section{Discussion}

The aim of this work was to investigate whether a biomimetic periosteum, namely tissue-engineered bone membrane composites constructed in vitro, could form bone tissue in the allogenic rabbit. 

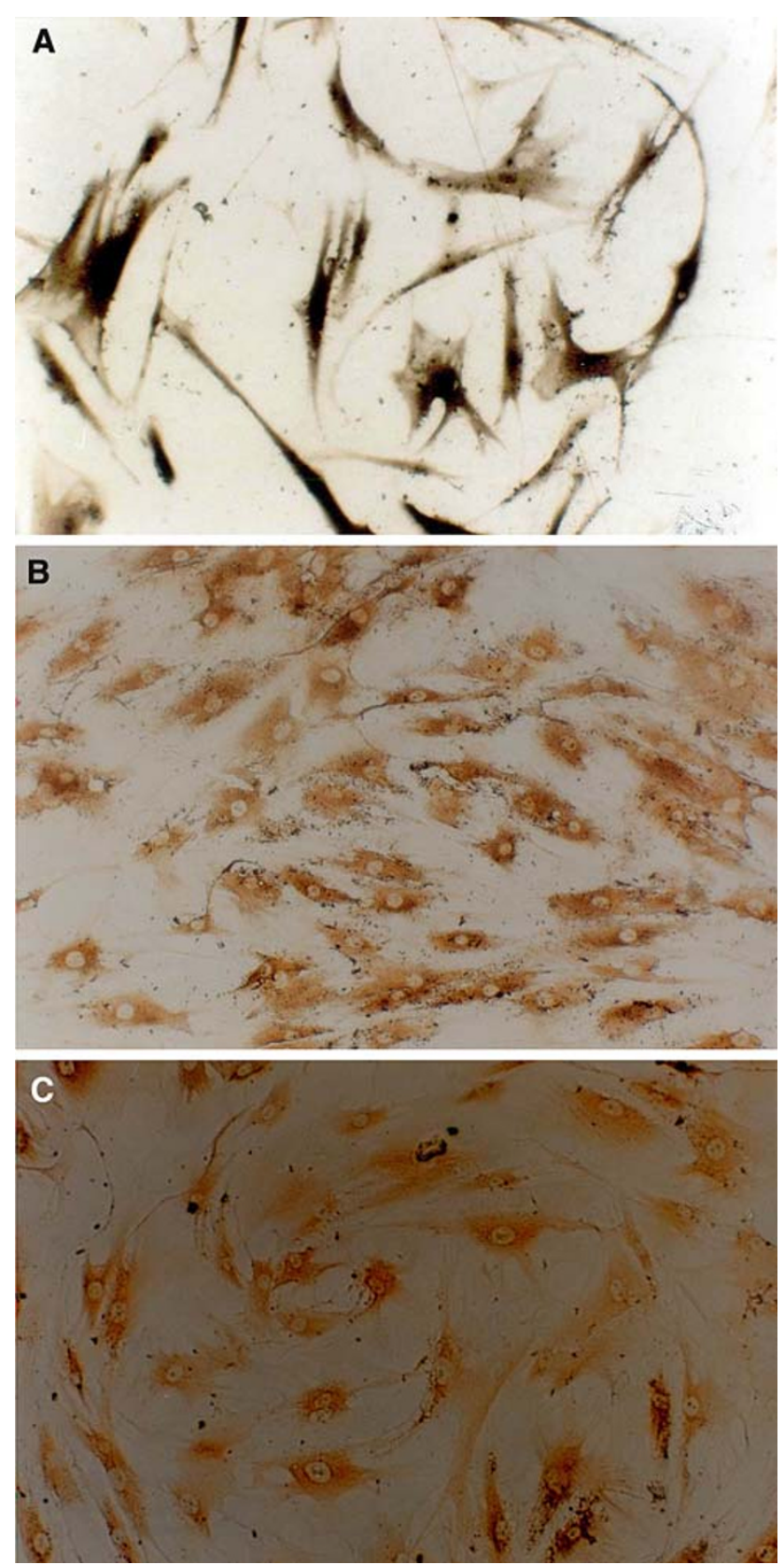

Fig. 2 MSCs induced for 7 days with a mixture of osteogenetic inducer of $50 \mathrm{mg} / \mathrm{L}$ ascorbic acid, $10 \mathrm{mmol} / \mathrm{L} \beta$-glycerophosphate and $10^{-8} \mathrm{~mol} / \mathrm{L}$ dexamethasone. Athough there was no obvious morphological change, microscopically, they already showed osteogenic differentiation. a Induced MSCs expressed large quantities of ALP intracellularly (ALP Gomori staining, $\times 100$ ). b, c show osteocalcin and I-collagen expression, respectively, confirmed by immunocytochemistry $(\mathrm{ABC} \times 100)$. All of these three phenotypes proved that the MSCs had differentiated into osteoblast

Although the seeding cells (MSCs or osteoblasts induced from MSCs) were allogenic, this study succeeded in forming new bone tissue without immunological rejection (as determined by histological examination). It was reported that MSCs have a highly reduced

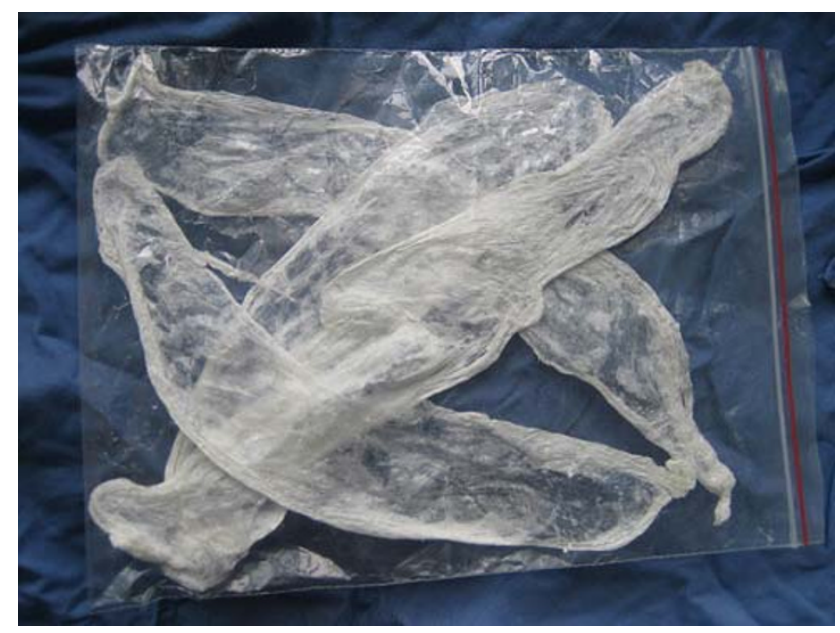

Fig. 3 Macroscopical view of scaffold (SIS straps sealed in a sterile plastic bag). Manufactured SIS is a white membrane-like biomaterial, $100 \pm 20 \mu \mathrm{m}$ in thickness, and the texture is just as flexible as natural bone membrane. This biomaterial was also easy to handle for clipping, sterile radiation and sealing preservation

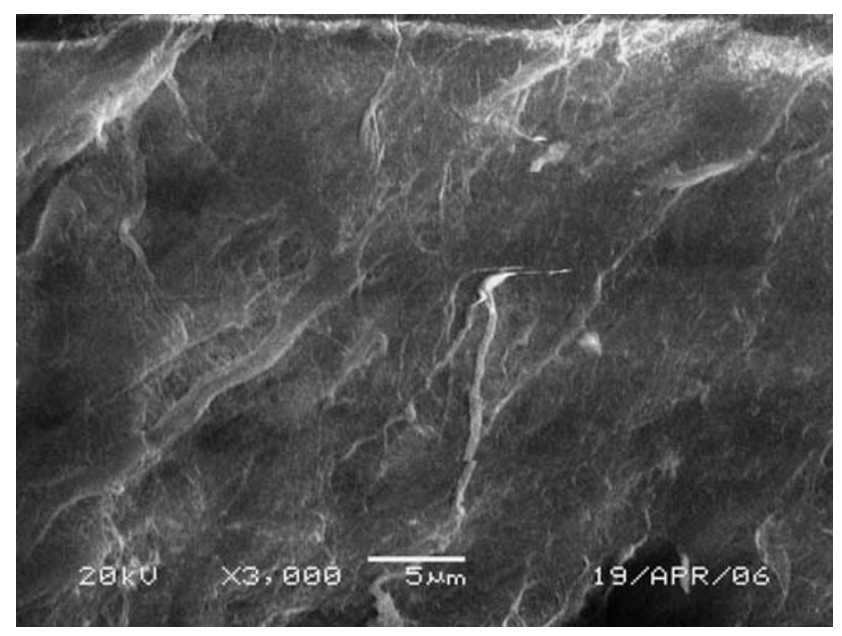

Fig. 4 SEM picture of the surface of SIS. Manufactured SIS show a velvet-like, broad surface with numerous fibre bundles, which form a variety of small, irregular porosities

immunoreactivity in allogenic transfer [22-24] and the results of this study confirm this again. In addition, it was shown that whilst the MSCs can survive and form bone tissue in an allogenic environment, the osteoblasts induced from MSCs still maintain the low-immunogenicity character of parent MSCs. This result holds potential for manufacturing tissue-engineered implants using allogenic bone marrow mesenchymal stem cells, which are free from immunological rejection. It also suggests that mature cellular lineages induced from these MSCs will have a low immunogenicity and opens the way for studying allogenic somatic mature cells transfers, an area which has always been impractical owing to the barrier of immunological rejection. 


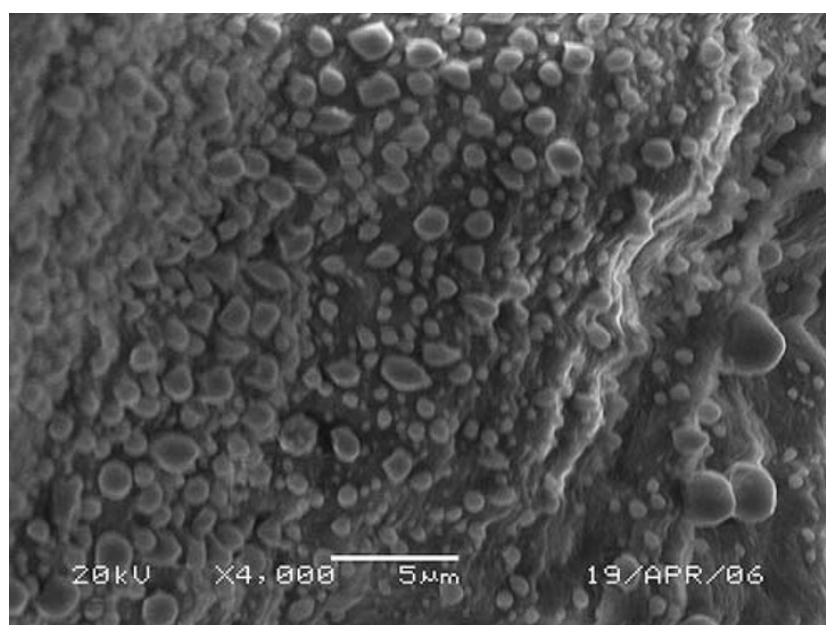

Fig. 5 SEM picture of tissue-engineered bone membrane after 5 days. The seeding cells adhered to the surface of SIS abundantly

Successful new bone formation, without evidence of immunological rejection, was also attributed to the SIS, which, despite being derived from a xenogeneous animal, has a low xenogeneic immunoreactivity, in accordance with published literature [25].

Two of the greatest challenges faced in bone tissue engineering is how to provide nutrition to the seeded cells in the early stages before the formation of new vessels and how to induce a natural capillary system into the implant composite later [13]. The thickness $(100 \pm 20 \mu \mathrm{m})$ of SIS can permit attached cells to survive early on through diffusion of nutrients from the interstitial fluid. The biodegraded leftovers of SIS may contribute to the formation of vascular tissues; it was noted in this study that the leftovers of SIS were accompanied with neovascules in cavities within the newly formed bone tissue. This suggests that SIS degradation is relevant to angiogenesis and the mechanism is thought to be linked to large amounts of vascular epithelial growth factor (VEGF) in the SIS [26-28].

Successful bone formation was observed in regions where the fragmental bone defect was treated with tissueengineered bone membrane constructed of either allogenic MSCs or with osteoblasts induced from allogenic MSCs. It is not possible, within the constraints of this study, to report on the relative efficacies of the different seeding cells in repairing bone defects, but the osteogenetic potential of allogenic tissue-engineered bone membrane in animals forms the basis for further study. The technique of bone regeneration through tissue engineering in this study is quite different from traditional ones, which have attempted to regenerate bone directly by constructing a 3-D implant [6-10]. This study has used a novel method of experimental bone formation (intramembranous osteogenesis) in a defect. Our results have asserted that the bone defect,
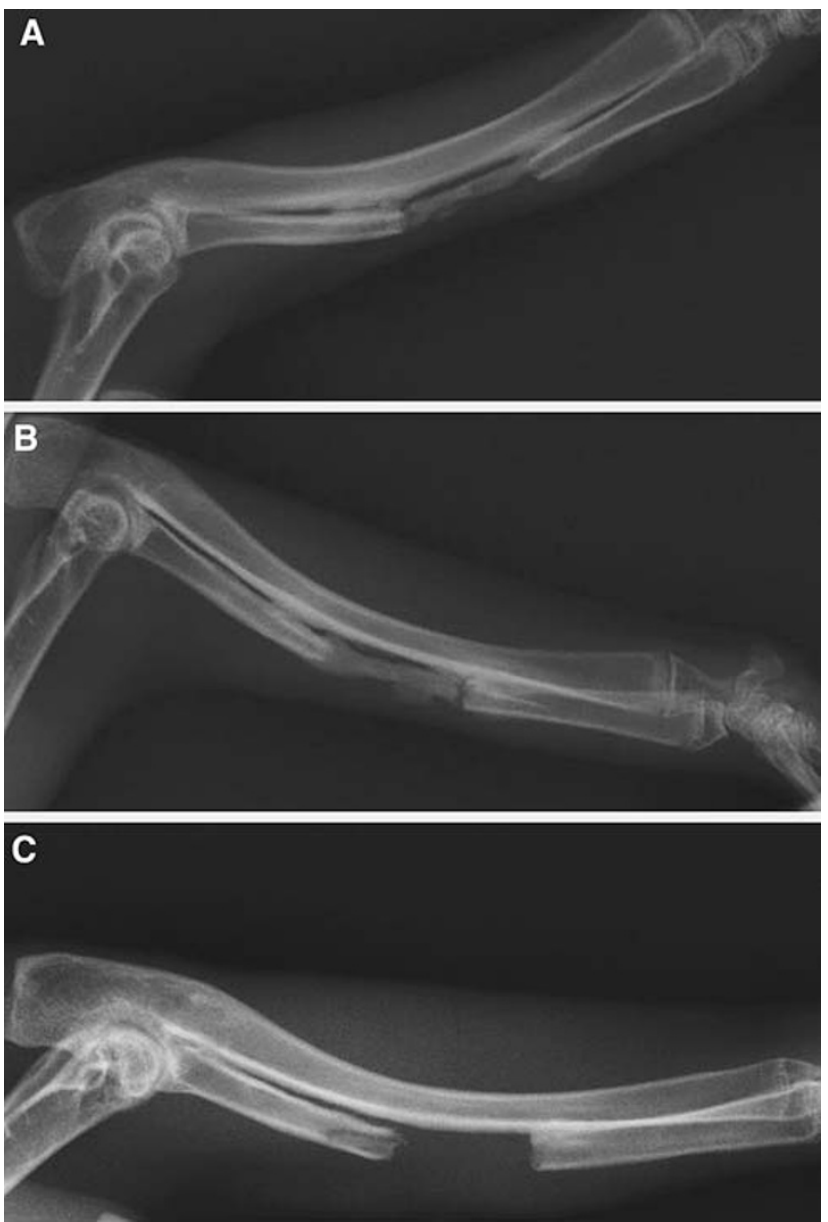

Fig. 6 Representative rabbit radial radiographs, 4 weeks after the implantation operation (red line shows the region of radial bone defect). a The segmental bone defect treated with tissue-engineered bone membrane M1(MSC + SIS) showed bone repairing. b The critical size bone defect treated with tissue-engineered bone membrane M2 (osteoblast induced from MSC + SIS) also showed bone repairing. c The segmental bone defect treated with SIS is empty under X-ray detection. These radiographs confirmed that critical bone defect can be repaired by tissue-engineered bone membrane, either constructed with seeding cells of allogenic MSCs or osteogenetic induced allogenic MSCs

even when large, may be repaired by tissue-engineered bone membrane efficiently because of naturally induced angiogenesis. The materials of this tissue-engineered composite, which are derived from the allogenic and the xenogenic sources are cheap and abundant and, therefore, may be much more practical for widespread clinical use.

This pilot qualitative study was conducted to observe the feasibility of an innovative scheme in bone tissue engineering. Although this has shown successful bone defect repair and offered a promising protocol to regenerate bone tissue, there are still several issues unanswered: for instance, the mechanism of immunogenicity against allogenic cells and xenogenic biomaterials; the long-term 


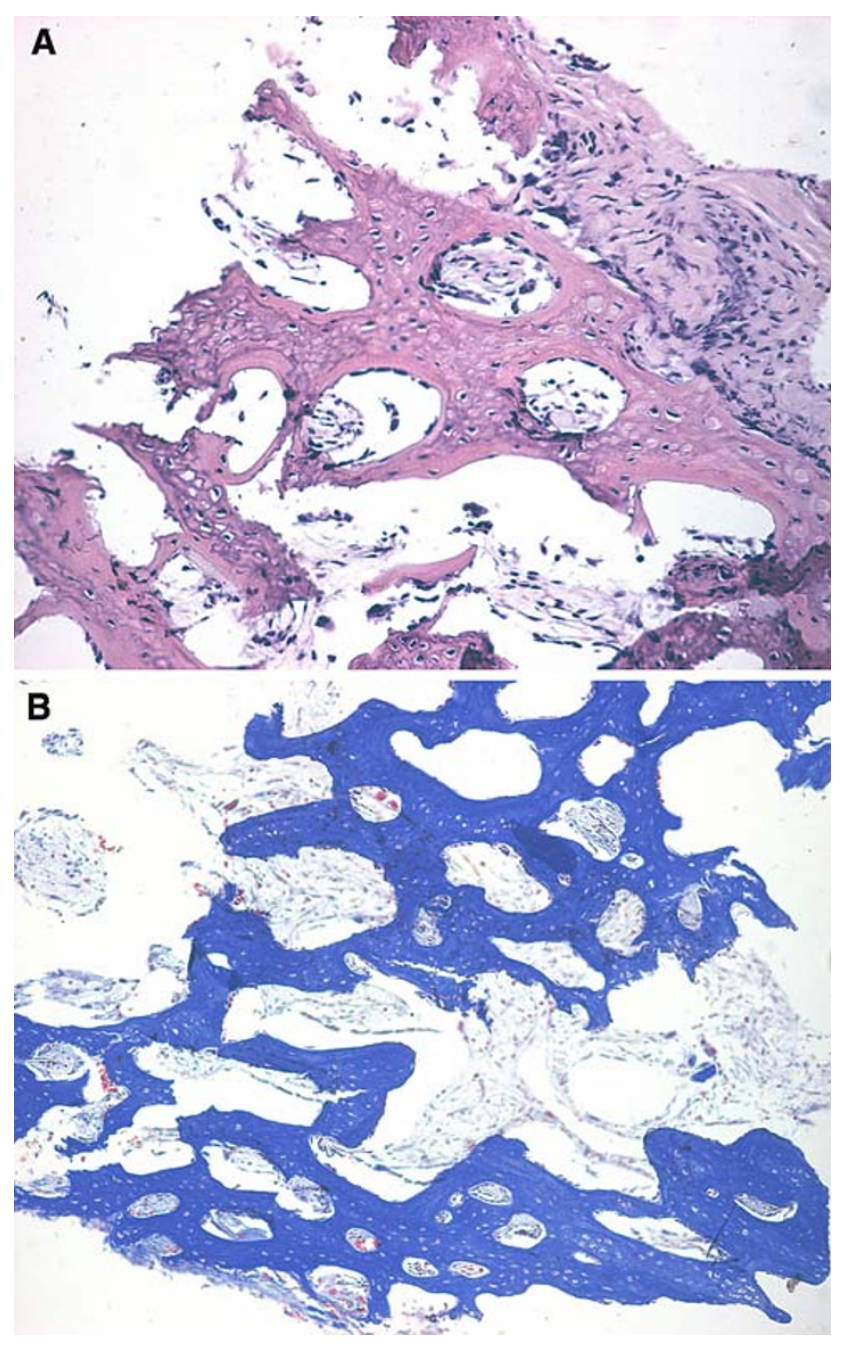

Fig. 7 Microscopically, histological detection showed new bone tissue formed by tissue-engineered bone membrane, and new bone tissue arranged irregularly around a lot of porosity, in which there were primary vessels and biodegraded leftovers of SIS. a HE staining $(\times 100)$; b Masson staining $(\times 100)$

behaviour of tissue-engineered bone; and quantitative aspects of this mechanism.

\section{Conclusion}

1. Tissue-engineered bone membrane, constructed by allogeneic seeding cells and xenogeneic bioderived materials, has osteogenetic potential and can repair fragmental bone defects successfully.

2. MSCs and osteoblasts induced from MSCs maintain a low immunogenicity in vivo within tissue from an allogenic animal.

3. SIS used as a scaffold is an ideal biomaterial for constructing tissue-engineered bone membrane because of its low immunogenicity and thin porous membrane structure.
4. Seeding cells attached on a thin membrane scaffold can survive because of the diffusion of nutrients from the interstitial fluid.

5. A vascular system can establish naturally in new bone tissue derived from tissue-engineered bone membrane.

Acknowledgments This work was supported by the National Natural Science Foundation of China (Project Grant No.30500133). The authors wish to thank J Liu, JS Wang and other members of the laboratory for their invaluable help.

\section{References}

1. Olivier G, Ralph M, Dietrich von S et al (2005) In vivo bone regeneration with injectable calcium phosphate biomaterial: a three-dimensional micro-computed tomographic, biomechanical and SEM study. Biomaterials 26:5444-5453

2. Parikh SN (2002) Bone graft substitutes: past, present, future. J Postgrad Med 48:142-148

3. Petite H, Viateau V, Bensaid W et al (2000) Tissue-engineered bone regeneration. Nat Biotechnol 18:959-963

4. Quarto R, M astrogiacomo M, Cancedda R et al (2001) Repair of large bone defects with the use of autologous bone marrow stromal cells. N Engl J Med 344(5):385-386

5. Shang Q, Wang Z, Liu W, Shi Y, Cui L, Cao Y (2001) Tissueengineered bone repair of sheep cranial defects with autologous bone marrow stromal cells. J Craniofac Surg 6:586-593

6. Shakesheff K, Cannizzaro S, Langer R (1998) Creating biomimetic microenvironments with synthetic polymer-peptide hybrid molecules. J Biomater Sci Polym Ed 9(5):507-518

7. Ishaug SL, Crane GM, Miller MJ, Yasko AW, Yaszemski MJ, Mikos AG (1997) Bone formation by three-dimensional stromal osteoblast culture in biodegradable polymer scaffolds. J Biomed Mater Res 36(1):17-28

8. Kotobuki N, Hirose M, Takakura Y, Ohgushi H (2004) Cultured autologous human cells for hard tissue regeneration: preparation and characterization of mesenchymal stem cells from bone marrow. Artif Organs 28:33-39

9. Turhani D, Watzinger E, Weissenbock M, Cvikl B, Thurnher D, Wittwer G, Yerit K, Ewers R (2005) Analysis of cell-seeded 3-dimensional bone constructs manufactured in vitro with hydroxyapatite granules obtained from red algae. J Oral Maxillofac Surg 63(5):673-681

10. Turhani D, Watzinger E, Weissenbock M, Yerit K, Cvikl B, Ewers R, Thurnher D (2005) Expression pattern of the chromosome 21 transcription factor Ets2 in cell-seeded three-dimensional bone constructs. J Biomed Mater Res A 73(4):445-455

11. Freed LE, Vunjak-Novakovic G (1998) Culture of organized cell communities. Adv Drug Deliv Rev 33:15

12. Hua Ye, Diganta B. Das, James T. Triffitt, et al (2006) Modelling nutrient transport in hollow fibre membrane bioreactors for growing three-dimensional bone tissue. J Membr Sci 272:169-178

13. Griffith LG, Naughton G (2002) Tissue engineering-current challenges and expanding opportunities. Science 295:1009

14. Mizuno H, Hata K, Kojima K, Bonassar LJ, Vacanti CA, Ueda M (2006) A novel approach to regenerating periodontal tissue by grafting autologous cultured periosteum. Tissue Eng 12(5):12271335

15. Vogelin E, Jones NF, Huang JI, Brekke JH, Lieberman JR (2005) Healing of a critical-sized defect in the rat femur with use of a vascularized periosteal flap, a biodegradable matrix, and bone morphogenetic protein. J Bone Joint Surg Am 87(6):1323-1331 
16. Kanou M,Ueno T, Kagawa T, Fujii T, Sakata Y, Ishida N, Fukunaga J, Sugahara T (2005) Osteogenic potential of primed periosteum graft in the rat calvarial model. Ann Plast Surg 54(1):71-78

17. Hattori K, Yoshikawa T, Takakura Y, Aoki H, Sonobe M, Tomita N (2005) Bio-artificial periosteum for severe open fracture-an experimental study of osteogenic cell/collagen sponge composite as a bio-artificial periosteum. Biomed Mater Eng 15(3):127-136

18. Aggarwal S, Pittenger MF (2005) Human mesenchymal stem cells modulate allogeneic immune cell responses. Blood 105:1815-1822

19. Pittenger MF, Mackay AM, Beck SC et al (1999) Multilineage potential of adult human mesenchymal stem cells. Science 284:143-147

20. Vats A, Tolley NS, Buttery LDK, Polak JM (2004) The stem cell in orthopaedic surgery. J Bone Joint Surg 86-B(2):159-64

21. Horwitz EM, Le Blanc K, Dominici M, Mueller I, SlaperCortenbach I, Marini FC et al (2005) Clarification of the nomenclature for MSC: the International Society for Cellular Therapy position statement. Cytotherapy 7(5):393-395

22. Le Blanc K, Tammik L, Sundberg B et al (2003) Mesenchymal stem cells inhibit and stimulate mixed lymphocyte cultures and mitogenic responses independently of the major histocompatibility complex. Scand J Immunol 57(1):11-20
23. Gotherstrom C, Ringden O, Tammik C et al (2004) Immunologic properties of human fetal mesenchymal stem cells. Am J Obstet Gynecol 190(1):239-245

24. Julius A (2003) Potian, Hana Aviv. Veto-like activity of mesenchymal stem cells: functional discrimination between cellular responses to alloantigens and recall antigens. J Immunol 171:34263434

25. Allman AJ, Mcpherson TB, Badylak SF et al (2001) Xenogeneic extracellular matrix grafts elicit a Th2-resistricted immune response. Transplantation 71(11):1631

26. Hodde JP, Record R, Liang HA et al (2001) Vascular endothelial growth factor in porcine-derived extracellular matrix. Endothelium 8(1): 11

27. Badylak SF, Liang HA, Record R et al (1999) Endothelial cell adherence to small intestinal submucosa: an acellular bioscaffold. Biomaterials 20(23-24):2257

28. Ishikawa K, Ueyama Y, Mano T et al (1999) Self-setting barrier membrane for guided tissue regeneration method: initial evaluation of alginate membrane made with sodium alginate and calcium chloride aqueous solutions. J Biomed Mater Res 47(2):111-115

29. Meinig RP (2002) Polylactide membranes in the treatment of segmental diaphyseal defects: animal model experiments in the rabbit raius, sheep tibia, Yucatan minipig radius, and goat tibia. Injury 33(Suppl 2):B-58-B-65 